\title{
Critical Bifurcation Surfaces of 3D Discrete Dynamics
}

\author{
MICHAEL SONIS* \\ Department of Geography, Bar-Ilan University, Ramat-Gan 52900, Israel
}

(Received 25 June 1999)

\begin{abstract}
This paper deals with the analytical representation of bifurcations of each 3D discrete dynamics depending on the set of bifurcation parameters. The procedure of bifurcation analysis proposed in this paper represents the 3D elaboration and specification of the general algorithm of the $n$-dimensional linear bifurcation analysis proposed by the author earlier. It is proven that 3D domain of asymptotic stability (attraction) of the fixed point for a given 3D discrete dynamics is bounded by three critical bifurcation surfaces: the divergence, flip and flutter surfaces. The analytical construction of these surfaces is achieved with the help of classical Routh-Hurvitz conditions of asymptotic stability. As an application the adjustment process proposed by T. Puu for the Cournot oligopoly model is considered in detail.
\end{abstract}

Keywords: Bifurcation surfaces, Routh-Hurvitz conditions, Cournot oligopoly

\section{INTRODUCTION}

The purpose of this paper is to construct the 3D analytical representation of the general procedure of linear bifurcation analysis developed by Sonis $(1993,1997)$. The bifurcation analysis describes the changes in the qualitative properties of the orbits on non-linear discrete dynamics under the changes of the (external) parameters of these dynamics.

The bifurcation phenomena are defined by the position of the boundaries of attraction of the fixed point. It will be proven further that the domain of attraction of the fixed point of 3D discrete dynamics is bounded by three critical bifurcation surfaces: the divergence surface corresponding to the case in which one of the eigenvalues of the
Jacobi matrix of the linear approximation of the dynamics equals to 1 ; the flip surface corresponding to the existence of the eigenvalue -1 , and the flutter surface corresponding to the pair of complex conjugated eigenvalues with absolute values equal to 1 . The crossing of these surfaces by the movement of the fixed point will generate the plethora of all possible bifurcation phenomena.

\section{3D LINEAR BIFURCATION ANALYSIS}

\subsection{Fixed Points of 3D Discrete Non-Linear Dynamics}

Let us consider 3D discrete dynamics represented by the following system of autonomous difference

*E-mail: sonism@mail.biu.ac.il. 
equations:

$$
\begin{aligned}
& x_{t+1}=F\left(x_{t}, y_{t}, z_{t} ; A\right) \quad\left(=F_{t}\right) \\
& y_{t+1}=G\left(x_{t}, y_{t}, z_{t} ; A\right) \quad\left(=G_{t}\right) \\
& z_{t+1}=H\left(x_{t}, y_{t}, z_{t} ; A\right) \quad\left(=H_{t}\right) ; \quad t=0,1,2, \ldots
\end{aligned}
$$

where $A=\left\{a_{1}, a_{2}, a_{3}, \ldots, a_{k}\right\}$ is a set of external bifurcation parameters and $F_{t}, G_{t}, H_{t}$ are the differentiable functions of $x_{t}, y_{t}, z_{t}$. For the fixed set $A$ all fixed points $(x, y, z)$ of the system (2.1) are given by the solutions of the system of algebraic equations

$$
\begin{gathered}
x=F(x, y, z ; A) ; \quad y=G(x, y, z ; A) ; \\
z=H(x, y, z ; A)
\end{gathered}
$$

\subsection{The Value of the Jacobi Matrix at a Fixed Point}

Let us now present in detail the $3 \mathrm{D}$ procedure of linear bifurcation analysis. First step of the analysis includes the construction of the Jacobi matrix of a linear approximation of the non-linear dynamics (2.1):

$$
J_{(t, t+1)}=\left[\begin{array}{lll}
\frac{\partial F_{t}}{\partial x_{t}} & \frac{\partial F_{t}}{\partial y_{t}} & \frac{\partial F_{t}}{\partial z_{t}} \\
\frac{\partial G_{t}}{\partial x_{t}} & \frac{\partial G_{t}}{\partial y_{t}} & \frac{\partial G_{t}}{\partial z_{t}} \\
\frac{\partial H_{t}}{\partial x_{t}} & \frac{\partial H_{t}}{\partial y_{t}} & \frac{\partial H_{t}}{\partial z_{t}}
\end{array}\right]
$$

and its value at the fixed point $(x, y, z)$ :

$$
J=\left[\begin{array}{lll}
\frac{\partial F}{\partial x} & \frac{\partial F}{\partial y} & \frac{\partial F}{\partial z} \\
\frac{\partial G}{\partial x} & \frac{\partial G}{\partial y} & \frac{\partial G}{\partial z} \\
\frac{\partial H}{\partial x} & \frac{\partial H}{\partial y} & \frac{\partial H}{\partial z}
\end{array}\right]
$$

where functions $F, G, H$ are results of substitution $\left(x_{t}, y_{t}, z_{t}\right) \rightarrow(x, y, z)$ in $F_{t}, G_{t}, H_{t}$ :

$$
\begin{aligned}
& F=F(x, y, z ; A)=\left[F_{t}\right]_{\substack{x_{t}=x \\
y_{t}=y \\
z_{t}=z}} \\
& G=G(x, y, z ; A)=\left[G_{t}\right]_{\substack{x_{t}=x \\
y_{t}=y \\
z_{t}=z}} \\
& H=H(x, y, z ; A)=\left[H_{t}\right]_{\substack{x_{t}=x \\
y_{t}=y \\
z_{t}=z}}
\end{aligned}
$$

The eigenvalues of the Jacobi matrix $J$ are the solutions of the characteristic polynomial equation:

$$
\mu^{3}+c_{1} \mu^{2}+c_{2} \mu+c_{3}=0
$$

where

$$
\begin{gathered}
-c_{1}=\operatorname{Tr} J=\frac{\partial F}{\partial x}+\frac{\partial G}{\partial y}+\frac{\partial H}{\partial z} \\
J=\left|\begin{array}{ll}
\frac{\partial F}{\partial x} & \frac{\partial F}{\partial y} \\
\frac{\partial G}{\partial x} & \frac{\partial G}{\partial y}
\end{array}\right|+\left|\begin{array}{ll}
\frac{\partial F}{\partial x} & \frac{\partial F}{\partial z} \\
\frac{\partial H}{\partial x} & \frac{\partial H}{\partial z}
\end{array}\right|+
\end{gathered} \mid
$$$$
c_{2}=\Delta J=\left|\begin{array}{ll}
\frac{\partial F}{\partial x} & \frac{\partial F}{\partial y} \\
\frac{\partial G}{\partial x} & \frac{\partial G}{\partial y}
\end{array}\right|+\left|\begin{array}{ll}
\frac{\partial F}{\partial x} & \frac{\partial F}{\partial z} \\
\frac{\partial H}{\partial x} & \frac{\partial H}{\partial z}
\end{array}\right|+\left|\begin{array}{cc}
\frac{\partial G}{\partial y} & \frac{\partial G}{\partial z} \\
\frac{\partial H}{\partial y} & \frac{\partial H}{\partial z}
\end{array}\right|
$$

\subsection{Domain of Attraction of the Fixed Point and its Routh-Hurwitz Inequalities}

If $\mu_{1}, \mu_{2}, \mu_{3}$ are the roots of the characteristic Eq. (2.6) then as well known:

$$
\begin{gathered}
c_{1}=-\left(\mu_{1}+\mu_{2}+\mu_{3}\right) ; \quad c_{2}=\mu_{1} \mu_{2}+\mu_{1} \mu_{3}+\mu_{2} \mu_{3} \\
c_{3}=-\mu_{1} \mu_{2} \mu_{3}
\end{gathered}
$$

By the von Neiman theorem the fixed point $(x, y, z)$ is asymptotically stable iff for all eigenvalues of the Jacobi matrix $J$ the condition holds:

$$
|\mu|<1
$$

Condition (2.11) defines the space of parameters $c_{1}, c_{2}, c_{3}$ (space of eigenvalues) the geometrical domain of asymptotic stability (domain of attraction). The analytical description of this domain can be given with the help of classical Routh-Hurwitz algorithm in the form of non-linear inequalities (see, Samuelson, 1983, pp. 435-437; Sonis, 1997). For the derivation of these inequalities, for 3D discrete dynamics we construct first the new 
parameters $b_{0}, b_{1}, b_{2}, b_{3}$ such that:

$b_{0}=1+c_{1}+c_{2}+c_{3} ; \quad b_{1}=3+c_{1}-c_{2}-3 c_{3}$

$b_{2}=3-c_{1}-c_{2}+3 c_{3} ; \quad b_{3}=1-c_{1}+c_{2}-c_{3}$

Further we construct the Routh-Hurwitz matrix

$$
\left[\begin{array}{ccc}
b_{1} & b_{3} & 0 \\
b_{0} & b_{2} & 0 \\
0 & b_{1} & b_{3}
\end{array}\right]
$$

and its main minors:

$$
\begin{gathered}
\Delta_{1}=b_{1} ; \quad \Delta_{2}=\left|\begin{array}{cc}
b_{1} & b_{3} \\
b_{0} & b_{2}
\end{array}\right|=b_{1} b_{2}-b_{0} b_{3} ; \\
\Delta_{3}=\left|\begin{array}{ccc}
b_{1} & b_{3} & 0 \\
b_{0} & b_{2} & 0 \\
0 & b_{1} & b_{3}
\end{array}\right|=b_{3} \Delta_{2}
\end{gathered}
$$

The classical condition of asymptotic stability are

$$
b_{0}>0 ; \quad \Delta_{1}>0 ; \quad \Delta_{2}>0 ; \quad \Delta_{3}>0
$$

which means that:

$$
\begin{gathered}
b_{0}>0 ; \quad b_{1}>0 ; \quad b_{2}>0 ; \quad b_{3}>0 \\
\Delta_{2}=b_{1} b_{2}-b_{0} b_{3}>0
\end{gathered}
$$

\subsection{Geometrical Structure of the Domain of Attraction: Critical Bifurcation Surfaces}

It is possible to see that the inequalities

$$
b_{0}>0 ; \quad b_{1}>0 ; \quad b_{2}>0 ; \quad b_{3}>0
$$

define in the space of eigenvalues $c_{1}, c_{2}, c_{3}$ the interior of the pyramid with the vertices

$$
\begin{aligned}
& A(-1,-1,1), B(1,-1,-1), \\
& C(3,3,1), D(-3,3,-1)
\end{aligned}
$$

such that the plane $b_{0}=0$ lies on the triangle $A D B$; the plane $b_{1}=0$ lies on the triangle $A C D$; the plane

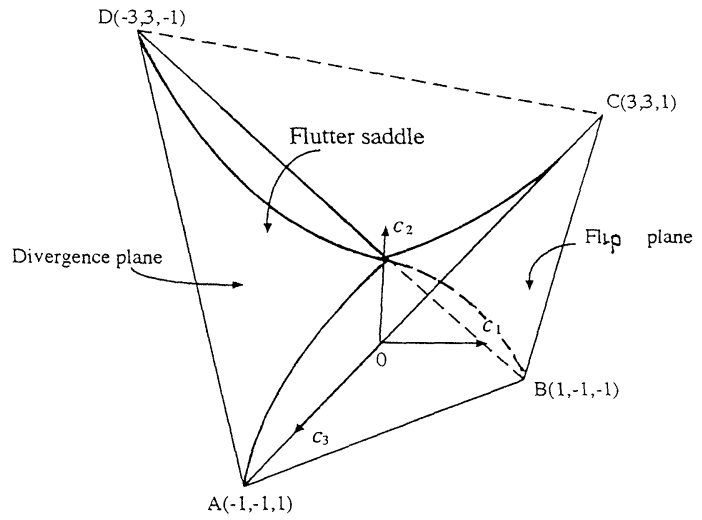

FIGURE 1 Domain of attraction of 3D discrete dynamics.

$b_{2}=0$ lies on the triangle $B C D$; and the plane $b_{3}=0$ lies on the triangle $A B C$ (see Fig. 1).

In addition, the inequality

$$
\Delta_{2}=b_{1} b_{2}-b_{0} b_{3}>0
$$

defines the part of the pyramid which includes the point $(0,0,0)$ and lies under the saddle

$$
\Delta_{2}=b_{1} b_{2}-b_{0} b_{3}=1-c_{2}+c_{1} c_{3}+c_{3}^{2}=0
$$

Thus, the domain of attraction of the fixed point $(x, y, z)$ is defined by three inequalities:

$$
\begin{aligned}
b_{0} & =1+c_{1}+c_{2}+c_{3}>0 \\
b_{3} & =1-c_{1}+c_{2}-c_{3}>0 \\
\Delta_{2} & =1-c_{2}+c_{1} c_{3}-c_{3}^{2}>0
\end{aligned}
$$

The boundaries of the domain of attraction are two planes,

$$
\begin{aligned}
& b_{0}=1+c_{1}+c_{2}+c_{3}=0 \\
& b_{3}=1-c_{1}+c_{2}-c_{3}=0
\end{aligned}
$$

and a saddle

$$
\Delta_{2}=1-c_{2}+c_{1} c_{3}-c_{3}^{2}=0
$$

The plane $b_{0}=0$ intersects the saddle by the sides $A D$ and $B D$; and the plane $b_{3}=0$ intersects the 
saddle by the sides $A C$ and $B C$; these two planes and the saddle will be called critical bifurcation surfaces.

\subsection{Real and Complex Eigenvalues in the Domain of Attraction}

It is important to note that the domain of attraction is divided into two parts by a saddle-like surface in such a manner that one part includes three real eigenvalues and the other one includes two complex conjugated eigenvalues and one real eigenvalue. For the construction of such a surface let us use the classical Thchirnhausen transformation:

$$
\mu=\lambda-\frac{c_{1}}{3}
$$

This transformation converts the characteristic polynomial Eq. (2.6) into

$$
\lambda^{3}+p \lambda+q=0
$$

where

$$
p=c_{2}-\frac{c_{1}^{2}}{3} ; \quad q=c_{3}-\frac{c_{1} c_{2}}{3}-\frac{2}{27} c_{1}^{3}
$$

The well-known classical condition for Eq. (2.25) (and same as for Eq. (2.6)) to have only real roots is the negativeness of the discriminant:

$$
\Delta=\left(\frac{p}{3}\right)^{3}+\left(\frac{q}{2}\right)^{2} \leq 0
$$

Thus, the surface

$$
\Delta=\left(\frac{p}{3}\right)^{3}+\left(\frac{q}{2}\right)^{2}=0
$$

is dividing the domain of attraction into the above mentioned two parts.

In the space of eigenvalues this surface has an equation:

$$
\Delta=\frac{c_{3}^{2}}{4}+\frac{c_{2}^{3}}{27}-\frac{c_{1} c_{2} c_{3}}{6}+\frac{c_{1}^{3} c_{3}}{27}-\frac{c_{1}^{2} c_{2}^{2}}{108}=0
$$

This surface includes the origin. Moreover the plane $b_{0}=0$ intersects this surface by the sides $A D$ and $B D$, and the plane $b_{3}=0$ intersects this surface by the sides $A C$ and $B C$. The coordinate plane $c_{1}=0$ intersects this surface by the cubic curve

$$
\frac{c_{3}^{2}}{4}+\frac{c_{2}^{3}}{27}=0
$$

the coordinate plane $c_{2}=0$ intersects this surface by the parabola:

$$
c_{3}=\frac{4}{27} c_{1}^{2}
$$

and the coordinate plane $c_{3}=0$ intersects this surface by the parabola:

$$
c_{2}=\frac{c_{1}^{2}}{4}
$$

\subsection{Structure of Bifurcations on the Critical Surfaces}

The formulae (2.10) imply that

$$
\begin{aligned}
& b_{0}=1+c_{1}+c_{2}+c_{3}=\left(1-\mu_{1}\right)\left(1-\mu_{2}\right)\left(1-\mu_{3}\right) \\
& b_{3}=1-c_{1}+c_{2}-c_{3}=\left(1+\mu_{1}\right)\left(1+\mu_{2}\right)\left(1+\mu_{3}\right)
\end{aligned}
$$

Thus, on the plane $b_{0}=0$ at least one of the eigenvalues is equal to 1, i.e., dynamics became divergent - this is a divergence plane. On the plane $b_{3}=0$ at least one of the eigenvalues is equal to -1 , i.e., dynamics became oscillatory, this is a flip plane. Each point on the flip triangle $A B C$ (see Fig. 1) corresponds to the two-periodic cycle, and the movement of the fixed point through it generates the Feigenbaum type periodic doubling sequence in three dimensions, leading to chaos (Feigenbaum, 1978).

It is possible to check that on the saddle (2.23)

$$
\begin{aligned}
\Delta_{2} & =1-c_{2}+c_{1} c_{3}-c_{3}^{2} \\
& =\left(1-\mu_{1} \mu_{2}\right)\left(1-\mu_{1} \mu_{3}\right)\left(1-\mu_{2} \mu_{3}\right)=0
\end{aligned}
$$


This equality defines the structure of bifurcations on the saddle in the following way. On the saddle all three absolute values of each eigenvalue are not more than 1 , since a saddle is the boundary of the domain of asymptotic stability and, moreover, on the saddle we have one real eigenvalue and two complex conjugate eigenvalues, say,

$$
\mu_{1,2}=R(\cos 2 \pi \Omega \pm \mathrm{i} \sin 2 \pi \Omega) ; \quad \mu_{3}=r
$$

The condition (2.34) means that

$$
\mu_{1} \mu_{2}=1
$$

Thus,

$$
\begin{gathered}
\left|\mu_{1}\right|=\left|\mu_{2}\right|=1, \quad R=1 ; \\
-1 \leq r \leq 1, \quad 0 \leq \Omega \leq 1
\end{gathered}
$$

Let us denote

$$
\alpha=2 \cos 2 \pi \Omega
$$

Obviously,

$$
-2 \leq \alpha \leq 2
$$

Equation (2.10) implies

$$
\begin{aligned}
& c_{1}=-\left(\mu_{1}+\mu_{2}+\mu_{3}\right)=-2 \cos 2 \pi \Omega-r=-\alpha-r \\
& c_{2}=\mu_{1} \mu_{2}+\mu_{1} \mu_{3}+\mu_{2} \mu_{3} 1+2 r \cos 2 \pi \Omega=1+\alpha r \\
& c_{3}=-\mu_{1} \mu_{2} \mu_{3}=-r
\end{aligned}
$$

If parameter $\alpha$ is fixed and $r$ is changing, then Eqs. (2.40) describe a straight line in the space of eigenvalues:

$c_{1}=-\alpha-r ; c_{2}=1+\alpha r ; c_{3}=-r ;-1 \leq r \leq 1$

This straight line is the straight line generator of the saddle, it lies on the saddle and intersects the side $A C$ in the point $(1-\alpha, 1-\alpha, 1)$ and the side $B D$ in the point $(-1-\alpha, 1+\alpha,-1)$ (see Fig. 2).

If $\Omega$ is a rational fraction: $\Omega=p / q$ then we have the so-called $q$-periodic resonances; between them

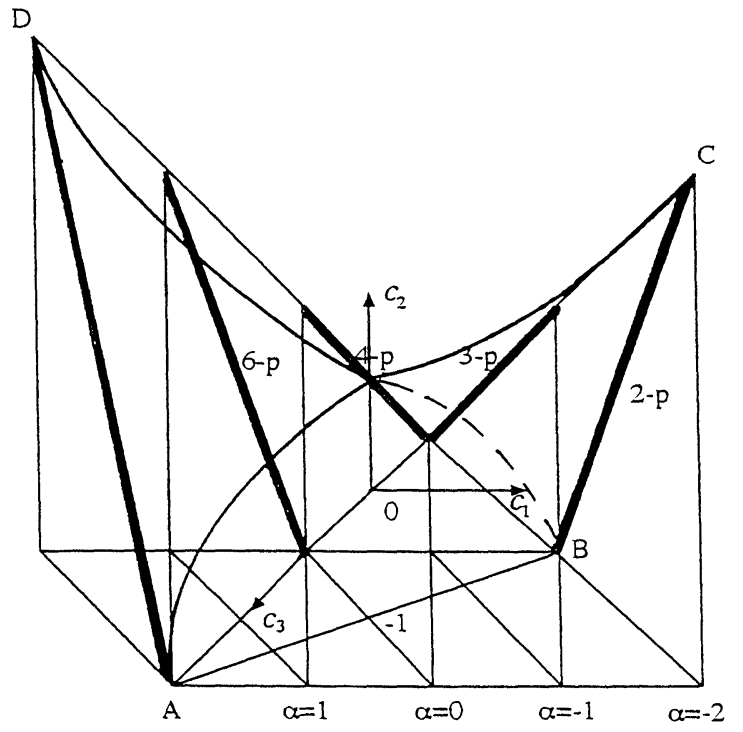

FIGURE 2 The segments of resonances for 3D discrete dynamics.

the fixed points of the strong 3-periodic (3-p) and 4-periodic (4- $p$ ) resonances corresponding to $\Omega=$ $1 / 3 ; \Omega=1 / 4$. Strong $3-p$ resonance corresponds to $\alpha=-1$ and lies on the segment of the straight line:

$c_{1}=1-r ; \quad c_{2}=1-r ; \quad c_{3}=-r ; \quad-1 \leq r \leq 1$

connecting the points $(2,2,1)$ and $(0,0,-1)$.

Strong 4- $p$ resonance corresponds to $\alpha=0$ and lies on the segment of the straight line:

$c_{1}=-r ; \quad c_{2}=1 ; \quad c_{3}=-r ; \quad-1 \leq r \leq 1$

connecting the points $(1,1,1)$ and $(-1,1,-1)$.

Other rational fractions represent fixed points of weak resonances. For example, 6- $p$ weak resonance corresponds to $\alpha=1$ and lies on the segment of the straight line:

$c_{1}=-1-r ; \quad c_{2}=1+r ; \quad c_{3}=-r ; \quad-1 \leq r \leq 1$

connecting the points $(0,0,1)$ and $(-2,2,-1)$. 
Table I represents the fixed points generating all $q$-periodic orbits for $q<12$.

If $\Omega$ is irrational, one obtains the quasi-periodic orbits.

In the following, we will call an inequality

$$
b_{0}=1+c_{1}+c_{2}+c_{3}>0
$$

the divergence inequality; the inequality

$$
b_{3}=1-c_{1}+c_{2}-c_{3}>0
$$

the flip inequality; the inequality

$$
\Delta_{2}=1-c_{2}+c_{1} c_{3}-c_{3}^{2}>0
$$

the flutter inequality.

\subsection{Special Case $c_{3}=0$; Domain of Attraction for 2D Dynamics}

If $c_{3}=0$ then the characteristic Eq. (2.6) will have a form:

$$
\mu^{2}+c_{1} \mu+c_{2}=0
$$

TABLE I Equilibria generating periodicity (till period 12)

\begin{tabular}{lcc}
\hline $\begin{array}{l}\text { Type of periodicity } \\
q\end{array}$ & $\begin{array}{c}\text { Arguments of eigenvalues } \\
\Omega=2 \pi p / q\end{array}$ & $\begin{array}{c}\text { Values of } \alpha \\
\alpha=2 \cos (2 \pi p / q)\end{array}$ \\
\hline 2 & $\pi$ & -2 \\
3 & $2 \pi / 3$ & -1 \\
4 & $\pi / 2$ & 0 \\
5 & $2 \pi / 5$ & 0.61803 \\
& $4 \pi / 5$ & -1.61803 \\
6 & $\pi / 3$ & 1 \\
7 & $2 \pi / 7$ & 1.24698 \\
& $4 \pi / 7$ & -0.44504 \\
& $6 \pi / 7$ & -1.80194 \\
8 & $\pi / 4$ & 1.41421 \\
9 & $2 \pi / 9$ & 1.53209 \\
& $4 \pi / 9$ & 0.34730 \\
& $8 \pi / 9$ & -1.87939 \\
10 & $\pi / 5$ & 1.61803 \\
& $3 \pi / 5$ & -0.61803 \\
11 & $2 \pi / 11$ & 1.68251 \\
& $4 \pi / 11$ & 0.83083 \\
& $6 \pi / 11$ & -0.28363 \\
& $8 \pi / 11$ & -1.30972 \\
12 & $10 \pi / 11$ & -1.91899 \\
& $\pi / 6$ & 1.73205 \\
\hline
\end{tabular}

which corresponds to the Jacobi matrix

$$
J_{(t, t+1)}^{(2)}=\left[\begin{array}{ll}
\frac{\partial F_{t}}{\partial x_{t}} & \frac{\partial F_{t}}{\partial y_{t}} \\
\frac{\partial G_{t}}{\partial x_{t}} & \frac{\partial G_{t}}{\partial y_{t}}
\end{array}\right]
$$

of 2D dynamics:

$$
\begin{array}{ll}
x_{t+1}=F\left(x_{t}, y_{t}, z_{t} ; A\right) & \left(=F_{t}\right) \\
y_{t+1}=G\left(x_{t}, y_{t}, z_{t} ; A\right) & \left(=G_{t}\right), \quad t=0,1,2, \ldots
\end{array}
$$

If

$$
x=F(x, y, z ; A) ; \quad y=G(x, y, z ; A)
$$

give the coordinates of the fixed point of the dynamics (2.50) we can calculate the value of the Jacobi matrix (2.49) in the fixed point $(x, y)$ from (2.51):

$$
J^{(2)}=\left[\begin{array}{ll}
\frac{\partial F}{\partial x} & \frac{\partial F}{\partial y} \\
\frac{\partial G}{\partial x} & \frac{\partial G}{\partial y}
\end{array}\right]
$$

where

$$
\begin{aligned}
& F=F(x, y, z ; A)=\left[F_{t}\right]_{\substack{x=x \\
y_{t}=y \\
t_{t}=y}} \\
& G=G(x, y, z ; A)=\left[G_{t}\right]_{\substack{x=x \\
y, y=y \\
t_{t}=y}}
\end{aligned}
$$

with the characteristic polynomial Eq. (2.48) where

$$
\begin{gathered}
-c_{1}=\operatorname{Tr} J^{(2)}=\frac{\partial F}{\partial x}+\frac{\partial G}{\partial y} \\
c_{2}=\operatorname{det} J^{(2)}=\left|\begin{array}{ll}
\frac{\partial F}{\partial x} & \frac{\partial F}{\partial y} \\
\frac{\partial G}{\partial x} & \frac{\partial G}{\partial y}
\end{array}\right|
\end{gathered}
$$

According to the Routh-Hurwitz procedure, the domain of stability of a fixed point for the 2D dynamics (2.47) is given by the system of inequalities (see Hsu, 1977; Sonis, 1993; 1996; 1997):

$$
-1 \pm c_{1}<c_{2}<1
$$


which represents the divergence, flip and flutter inequalities:

$$
\begin{aligned}
& b_{0}=1+c_{1}+c_{2}>0 ; \quad b_{3}=1-c_{1}+c_{2}>0 ; \\
& \Delta_{2}=1-c_{2}>0
\end{aligned}
$$

Figure 3 describes the domain of attraction, which is the triangle KLM in the space of eigenvalues $\left\{c_{1}, c_{2}\right\}$ with vertices

$$
K(-2,1), L(2,1), M(0,-1)
$$

The sides of the triangle of stability are defined by the following straight lines, the divergence boundary:

$$
1+c_{1}+c_{2}=0, \quad \text { or } \quad \operatorname{Tr} J^{(2)}=\operatorname{det} J^{(2)}+1
$$

the flip boundary:

$$
1+c_{1}-c_{2}=0, \quad \text { or } \quad-\operatorname{Tr} J^{(2)}=\operatorname{det} J^{(2)}+1
$$

and the flutter boundary:

$$
c_{2}=1, \quad \text { or } \quad \operatorname{det} J^{(2)}=1
$$

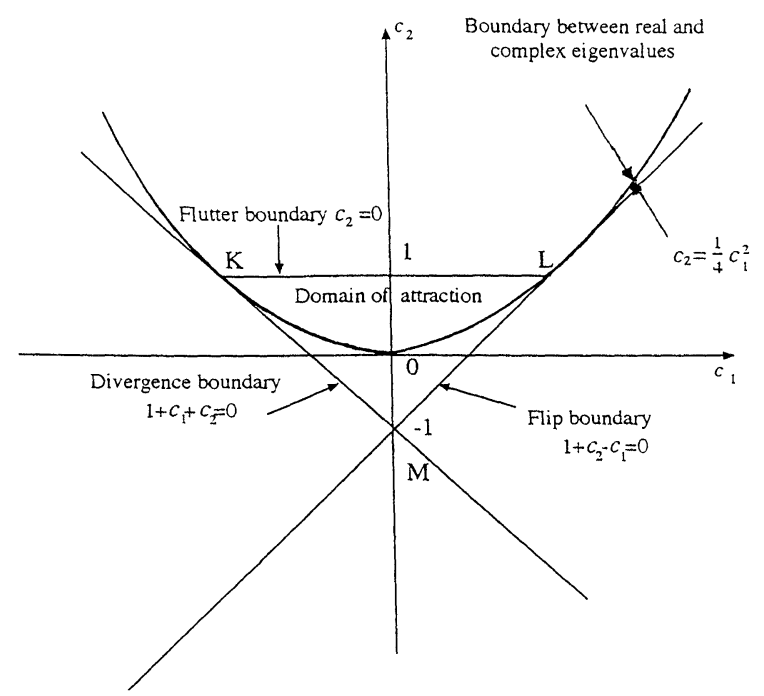

FIGURE 3 Domain of attraction of 2D discrete dynamics.
This domain (2.56) can be easily obtained from (2.21), putting in (2.21) $c_{3}=0$. Geometrically this means that the domain of stability of fixed point for $2 \mathrm{D}$ dynamics is the section of $3 \mathrm{D}$ domain of stability with the help of the coordinate plane $c_{3}=0$. The segment $\mathrm{KL}$ represents all flutter bifurcations of $2 \mathrm{D}$ dynamics. The $3 \mathrm{D}$ bifurcation segments (2.40) touch the flutter segment if $r=0$ and we have all the bifurcations corresponding to $\alpha=2 \cos 2 \pi \Omega$ in the points

$$
c_{1}=-\alpha ; \quad c_{2}=1 ; \quad c_{3}=0 ; \quad-2 \leq \alpha \leq 2
$$

So, Table I also describes the periodicities on flutter segment KL.

Special Case $c_{1}=0$ : A Dynamics without the SelfInfluence

The 3D dynamics without self-influence have a form:

$$
\begin{aligned}
& x_{t+1}=F\left(y_{t}, z_{t} ; A\right) \\
& y_{t+1}=G\left(x_{t}, z_{t} ; A\right) \\
& z_{t+1}=H\left(x_{t}, y_{t} ; A\right)
\end{aligned}
$$

where $x_{t+1}\left(y_{t+1}, z_{t+1}\right)$ does not explicitly depend on $x_{t}\left(y_{t}, z_{t}\right)$. In this case $c_{1}=0$ and the domain of stability of equilibria is described by divergence, flip and flutter inequalities:

$$
\begin{aligned}
& b_{0}=1+c_{2}+c_{3}>0 ; \quad b_{3}=1+c_{2}-c_{3}>0 ; \\
& \Delta_{2}=1-c_{2}-c_{3}^{2}>0
\end{aligned}
$$

Figure 4 describes the domain of stability in the space of the parameters $c_{2}, c_{3}$, whose boundaries are the flutter parabola $1-c_{2}=c_{3}^{2}$ and the divergence and flip straight lines: $1+c^{2}= \pm c_{3}$

The flutter segments (2.40) touches the flutter parabola if $r=-\alpha$ in the points:

$$
\begin{aligned}
c_{1} & =0 ; \quad c_{2}=1-\alpha^{2} ; \quad c_{3}=\alpha ; \\
& -1 \leq r=-\alpha \leq 1
\end{aligned}
$$

Table I indicates the impossible periodicities 8 and 12 where $\alpha>1$. 


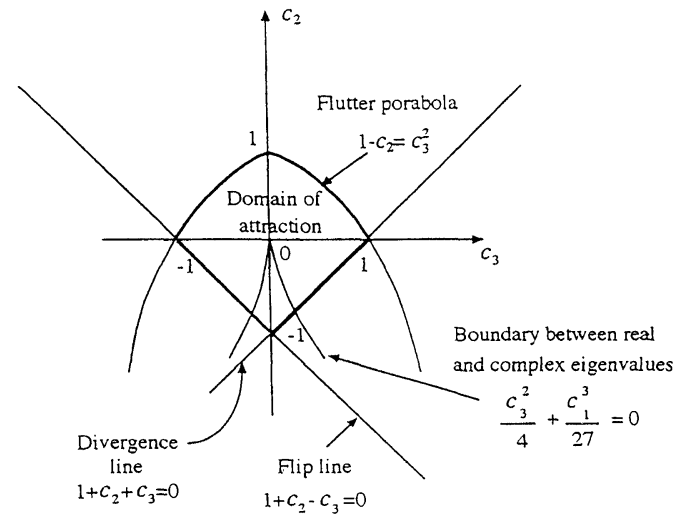

FIGURE 4 Domain of attraction of 3D non-self-influence discrete dynamics.

\section{COURNOT DYOPOLY AND TRIOPOLY: BIFURCATION ANALYSIS OF T. PUU ITERATIVE DYNAMICS}

\subsection{Dyopoly with Adjustment}

In his important book "Nonlinear Economic Dynamics" Swedish economist Tonu Puu introduced iterative process which leads two oligopolists to their Cournot equilibrium (see Puu, 1997, Chap. 5). T. Puu considered the following iteration process:

$$
\begin{aligned}
& x_{t+1}=x_{t}+\lambda\left(\sqrt{\frac{y_{t}}{a}-y_{t}-x_{t}}\right) \\
& y_{t+1}=y_{t}+\mu\left(\sqrt{\frac{x_{t}}{b}-x_{t}-y_{t}}\right)
\end{aligned}
$$

where $x_{t}, y_{t}$ are the supplies at time $t$ of two competitors in a duopoly, $a, b$ are their constant marginal costs, $\lambda, \mu$ are the adjustment speeds. Here it is assumed that $a, b>0 ; 0 \leq \lambda, \mu \leq 1$. The fixed point $(x, y)$ of this iteration dynamics satisfies the system of algebraic equations:

$$
\begin{aligned}
& x=x+\lambda\left(\sqrt{\frac{y}{a}-y-x}\right) \\
& y=y+\mu\left(\sqrt{\frac{x}{b}-x-y}\right)
\end{aligned}
$$

with a non-zero solution:

$$
x=\frac{b}{(a+b)^{2}} ; \quad y=\frac{a}{(a+b)^{2}}
$$

which is the Cournot equilibrium.

The Jacobi approximation matrix for the dynamics (3.1) is

$$
J_{t, t+1}=\left[\begin{array}{cc}
1-\lambda & \lambda\left(\frac{1}{2 \sqrt{a y}}-1\right) \\
\mu\left(\frac{1}{2 \sqrt{b x}}-1\right) & 1-\mu
\end{array}\right]
$$

In the Cournot equilibrium this matrix becomes

$$
J=\left[\begin{array}{cc}
1-\lambda & \frac{\lambda(b-a)}{2 a} \\
\frac{\mu(a-b)}{2 b} & 1-\mu
\end{array}\right]
$$

Therefore, from (2.51)

$$
-c_{1}=\operatorname{Tr} J=2-(\lambda+\mu)
$$

and from $(2.52)$

$$
c_{2}=\operatorname{det} J=(1-\lambda)(1-\mu)+\lambda \mu \frac{(a-b)^{2}}{4 a b}
$$

The divergence inequality $1+c_{1}+c_{2}<0$ becomes

$$
\begin{aligned}
1- & {[2-(\lambda+\mu)]+(1-\lambda)(1-\mu) } \\
+ & \lambda \mu \frac{(a-b)^{2}}{4 a b}>0
\end{aligned}
$$

which implies

$$
\lambda \mu \frac{(a+b)^{2}}{4 a b}>0
$$

which is always true for positive $a, b$ and $\lambda, \mu$, i.e. there are no situations in which iterative dynamics (3.1) diverges.

The flip inequality $1-c_{1}+c_{2}<0$ becomes

$$
\begin{aligned}
1+ & {[2-(\lambda+\mu)]+(1-\lambda)(1-\mu) } \\
+ & \lambda \mu \frac{(a-b)^{2}}{4 a b}>0
\end{aligned}
$$


or

$$
\lambda \mu \frac{(a+b)^{2}}{4 a b}>-2[2-(\lambda+\mu)]
$$

This inequality is always true, since the left side of (3.11) is positive and the right side is negative.

Thus, the domain of stability of the Cournot equilibrium is defined only by flutter inequality $\Delta_{2}=1-c_{2}>0$ which gives

$$
(1-\lambda)(1-\mu)+\lambda \mu \frac{(a-b)^{2}}{4 a b}<1
$$

or

$$
\frac{(a+b)^{2}}{4 a b}<\frac{1}{\lambda}+\frac{1}{\mu}
$$

Let us introduce the ratio of the marginal costs

$$
\frac{b}{a}=k
$$

and let

$$
\frac{1}{\lambda}+\frac{1}{\mu}=\Lambda
$$

Obviously, $k>0$ and $\Lambda>2$. Then, the domain of stability of the Cournot equilibrium will be

$$
\frac{(1+k)^{2}}{4 k}<\Lambda
$$

or

$$
k^{2}-2 k(2 \Lambda-1)+1<0
$$

which means that

$$
k_{1}<k<k_{2}
$$

where $k_{1}, k_{2}>0$ are the (positive) roots

$$
k_{1,2}=(2 \Lambda-1) \mp 2 \sqrt{\Lambda(\Lambda-1)}
$$

of the quadratic equation

$$
k^{2}-2 k(2 \Lambda-1)+1=0
$$

presenting the flutter boundary for the Cournot equilibrium.

Obviously, these roots are reciprocal, since we can use the reciprocal ratio $k=a / b$.

The character of bifurcations in these roots are the same and is defined by the value of $\alpha$ from (2.35) (see also Table I):

$\alpha=2 \cos 2 \pi \Omega=\operatorname{Tr} J=2-(\lambda+\mu)>0$

Few examples are of special interest: if the adjustments $\lambda, \mu$ are unitary, then $\alpha=0$ and we have (see, Section 2.5) 4- $p$ cycle starting the Feigenbaun double-periodic way to chaos; if $\lambda+\mu=1$, then $\alpha=1$ and we have 6- $p$ cycle bifurcations. Moreover, from (3.20) $\alpha>0$, thus, Table I indicates that the duopoly adjustment dynamics cannot have the 3-p bifurcation (with $\alpha=-1$ ), but it can have the 5- $p$ cycle corresponding to $\alpha=0.61803$, i.e., $\lambda+\mu=1.38157$.

\subsection{Cournot Equilibrium in Triopoly with Adjustment}

The case of triopoly can be considered analogically.

Tonu Puu introduced iterative process which leads three oligopolists to their Cournot equilibrium (see Puu, 1997, Chap. 5):

$$
\begin{aligned}
& x_{t+1}=\sqrt{\frac{y_{t}+z_{t}}{a}}-y_{t}-z_{t} \\
& y_{t+1}=\sqrt{\frac{z_{t}+x_{t}}{b}}-x_{t}-z_{t} \\
& z_{t+1}=\sqrt{\frac{x_{t}+y_{t}}{c}}-x_{t}-y_{t}
\end{aligned}
$$

This dynamics is non-self influence dynamics (see (2.7)) and its Cournot equilibrium

$$
\begin{gathered}
x=\frac{2(b+c-a)}{(a+b+c)^{2}} ; \quad y=\frac{2(a+c-b)}{(a+b+c)^{2}} ; \\
z=\frac{2(a+b-c)}{(a+b+c)^{2}}
\end{gathered}
$$


corresponds to the points in the $2 \mathrm{D}$ eigenvalue space (see Fig. 4). The domain of stability of this Cournot equilibrium can be easily calculated from the value of the Jacobi approximation matrix at the Cournot equilibrium

$$
\begin{aligned}
J & =\left[\begin{array}{ccc}
0 & \frac{b+c-3 a}{4 a} & \frac{b+c-3 a}{4 a} \\
\frac{c+a-3 b}{4 b} & 0 & \frac{c+a-3 b}{4 b} \\
\frac{a+b-3 c}{4 c} & \frac{a+b-3 c}{4 c} & 0
\end{array}\right] \\
& =\left[\begin{array}{ccc}
0 & k_{1} & k_{1} \\
k_{2} & 0 & k_{2} \\
k_{3} & k_{3} & 0
\end{array}\right]
\end{aligned}
$$

which gives (see (2.8) and (2.9))

$$
\begin{aligned}
-c_{1} & =\operatorname{Tr} J=0 \\
c_{2} & =\Delta J=-k_{1} k_{2}-k_{1} k_{3}-k_{2} k_{3} \\
-c_{3} & =\operatorname{det} J=-2 k_{1} k_{2} k_{3}
\end{aligned}
$$

In this case the domain of stability of Cournot equilibria is described by divergence, flip and flutter inequalities:

$$
\begin{aligned}
& b_{0}=1+c_{2}+c_{3}>0 ; \quad b_{3}=1+c_{2}-c_{3}>0 ; \\
& \Delta_{2}=1-c_{2}-c_{3}^{2}>0
\end{aligned}
$$

Figure 4 describes the domain of stability in the space of the parameters $c_{2}, c_{3}$, whose boundaries are the flutter parabola $1-c_{2}=c_{3}^{2}$ and the divergence and flip straight lines is $1+c_{2}= \pm c_{3}$. In the terms of $k_{1}, k_{2}, k_{3}$ the domain of structural stability of Cournot equilibrium is given by system of inequalities:

$$
1-4 k_{1}^{2} k_{2}^{2} k_{3}^{2}<k_{1} k_{2}+k_{1} k_{3}+k_{2} k_{3}<1 \pm 2 k_{1} k_{2} k_{3}
$$

Here the divergence surface is

$$
1-k_{1} k_{2}-k_{1} k_{3}-k_{2} k_{3}+2 k_{1} k_{2} k_{3}=0
$$

The two periodic flip bifurcations are "sitting" on the flip bifurcation surface:

$$
k_{1} k_{2}+k_{1} k_{3}+k_{2} k_{3}+2 k_{1} k_{2} k_{3}=1
$$

and the flutter periodic and quasi-periodic bifurcations belongs to flutter parabola $1-c_{2}=c_{3}^{2}$ generating the flutter bifurcation surface

$$
1+k_{1} k_{2}+k_{1} k_{3}+k_{2} k_{3}=4 k_{1}^{2} k_{2}^{2} k_{3}^{2}
$$

The character of flutter bifurcations is defined (see (2.8)) by

$$
2 \cos 2 \pi \Omega=\alpha=c_{3}=2 k_{1} k_{2} k_{3}
$$

or

$$
\Omega=\frac{1}{2 \pi} \arccos k_{1} k_{2} k_{3}
$$

The rational values of $\Omega$ give the periodic flutter bifurcations. As is indicated in (2.7) the triopoly cannot have 8 and 12 periodic bifurcations (cf. Table I). The irrational values of $\Omega$ give the quasiperiodic bifurcations.

\subsection{The "Virtual" Duopoly}

The "virtual" duopoly is the special case of triopoly with two identical competitors (see Puu, 1997, Chap. 5).

Thus, we can obtain from (3.22) the following 2D dynamics:

$$
\begin{aligned}
& x_{t+1}=\sqrt{\frac{2 y_{t}}{a}}-2 y_{t} \\
& y_{t+1}=\sqrt{\frac{x_{t}+y_{t}}{b}}-x_{t}-y_{t}
\end{aligned}
$$

with the corresponding Cournot equilibrium:

$$
x=\frac{2(2 b-a)}{(a+2 b)^{2}} ; \quad y=\frac{2 a}{(a+2 b)^{2}}
$$

The value of the corresponding Jacobi approximation matrix at the Cournot equilibrium (3.34) is

$$
J^{(2)}=\left[\begin{array}{cc}
0 & \frac{2 b-3 a}{2 a} \\
\frac{a-2 b}{4 b} & \frac{a-2 b}{4 b}
\end{array}\right]
$$


with

$$
\begin{aligned}
& -c_{1}=\operatorname{Tr} J^{(2)}=\frac{a-2 b}{4 b}=\frac{1-2 k}{4 k} \\
& c_{2}=\operatorname{det} J^{(2)}=\frac{(a-2 b)(3 a-2 b)}{8 a b} \\
& =\frac{(1-2 k)(3-2 k)}{8 k}
\end{aligned}
$$

where

$$
\frac{b}{a}=k
$$

is the ratio of the marginal costs.

The divergence inequality $1+c_{1}+c_{2}>0$ gives:

$$
1+\frac{2 k-1}{4 k}+\frac{(1-2 k)(3-2 k)}{8 k}>0
$$

which is equivalent to $(1+2 k)^{2}>0$ and thus holds always, so the adjustment dynamics cannot diverge. The flip inequality $1-c_{1}+c_{2}>0$ gives:

$$
1-\frac{2 k-1}{4 k}+\frac{(1-2 k)(3-2 k)}{8 k}>0
$$

which is equivalent to $5-4 k+4 k^{2}=4+$ $(1-2 k)^{2}>0$ and holds always, so the adjustment dynamics cannot flip. The flutter inequality $c_{2}<1$ gives:

$$
\frac{(1-2 k)(3-2 k)}{8 k}<1
$$

which is equivalent to

$$
3-16 k+4 k^{2}<0
$$

which means that

$$
\bar{k}_{1}<k<\bar{k}_{2}
$$

where

$$
\bar{k}_{1,2}=2 \pm \frac{\sqrt{13}}{2}
$$

are the roots of the quadratic equation

$$
4 k^{2}-16 k+3=0
$$

presenting the flutter boundary for the Cournot equilibrium. Thus, the domain of stability of the Cournot equilibrium (3.34) is the open segment $\left(\bar{k}_{1}, \bar{k}_{2}\right)=(2-(\sqrt{13} / 2), 2+(\sqrt{13} / 2))$. The end points of this segment presents the bifurcation points. Their nature is described by

$$
2 \cos 2 \pi \Omega=\alpha=\operatorname{Tr} J^{(2)}=\frac{1-2 k_{1,2}}{4 k_{1,2}}=\frac{1 \pm \sqrt{13}}{6}
$$

So, if $k$ approaches the value (3.44) then the attraction of the Cournot equilibrium (3.34) exchanged by the quasi-periodicity defined by the irrational

$$
\Omega=\frac{1}{2 \pi} \arccos \frac{1 \pm \sqrt{13}}{6}
$$

\section{References}

Feigenbaum, M. (1978) "Qualitative universality for a class of nonlinear transformations," Journal of Statistical Physics, 19: 25-52.

Hsu, C.S. (1977) "On non-linear parametric excitation problem," Advances in Applied Mathematics, 17: 245-301.

Samuelson, P.A. (1983) Foundations of Economic Analysis. Harvard Economic Studies, Vol. 80, Harvard University Press: Cambridge.

Sonis, M. (1993) "Behavior of iterational processes near the boundary of stability domain with applications to the sociospatial relative dynamics," in M. Drachlin and E. Litsin (Eds.) Functional-Differential Equations, Israel Seminar 1: 198-277.

Sonis, M. (1996) "Once more on Henon map: analysis of bifurcations." Chaos, Solitons \& Fractals, 7(12): 2215-2234.

Sonis, M. (1997) "Linear bifurcation analysis with applications to relative socio-spatial dynamics." Discrete Dynamics in Nature and Society, 1(1): 45-56.

Puu, T. (1997) Nonlinear Economic Dynamics. Fourth, completely revised and enlarged edition, Springer: Berlin, Heidelberg, New York. 


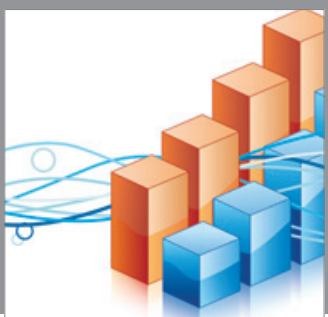

Advances in

Operations Research

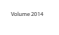

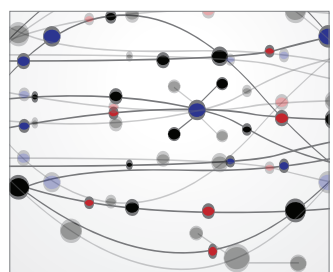

\section{The Scientific} World Journal
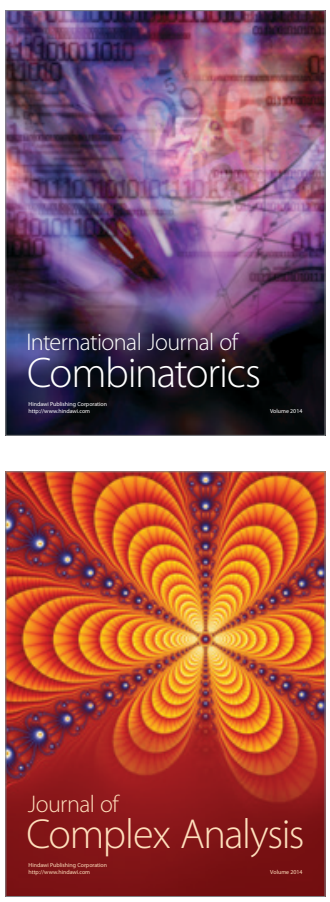

International Journal of

Mathematics and

Mathematical

Sciences
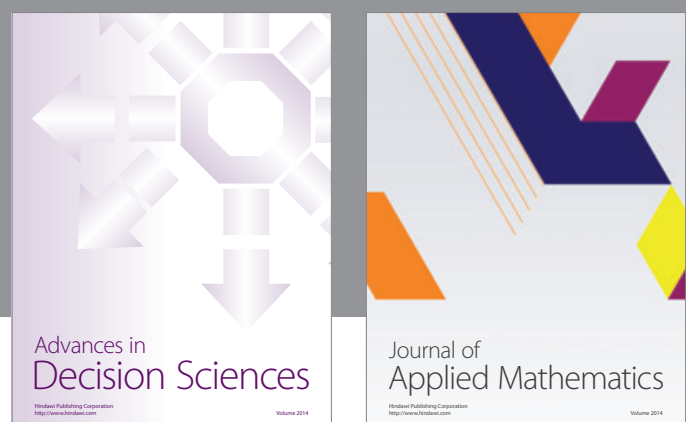

Journal of

Applied Mathematics
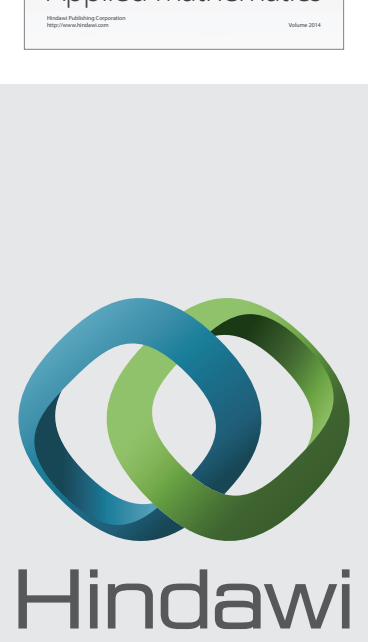

Submit your manuscripts at http://www.hindawi.com
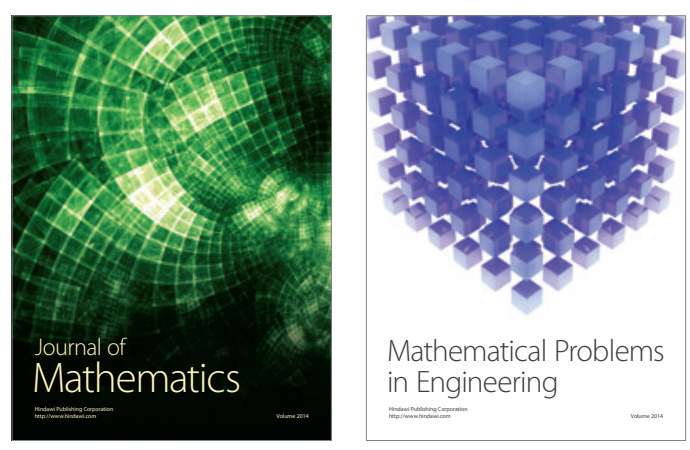

Mathematical Problems in Engineering
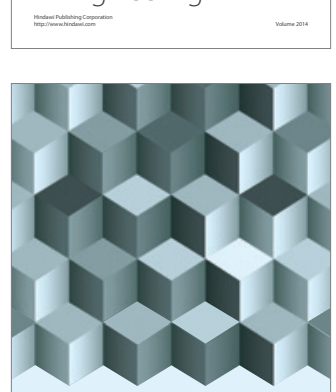

Journal of

Function Spaces
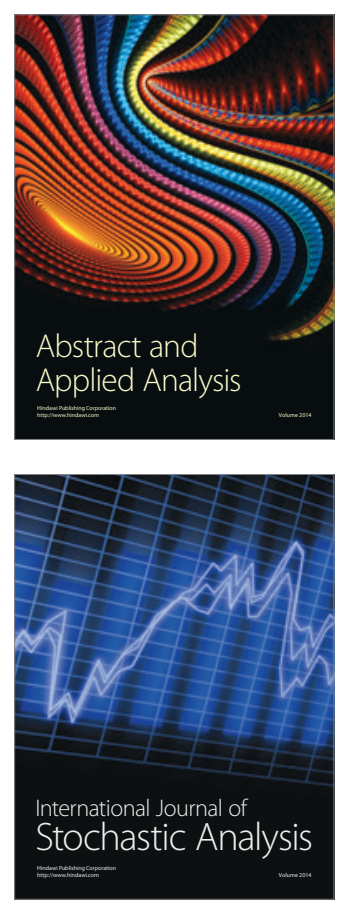

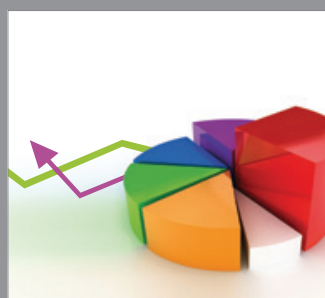

ournal of

Probability and Statistics

Promensencen
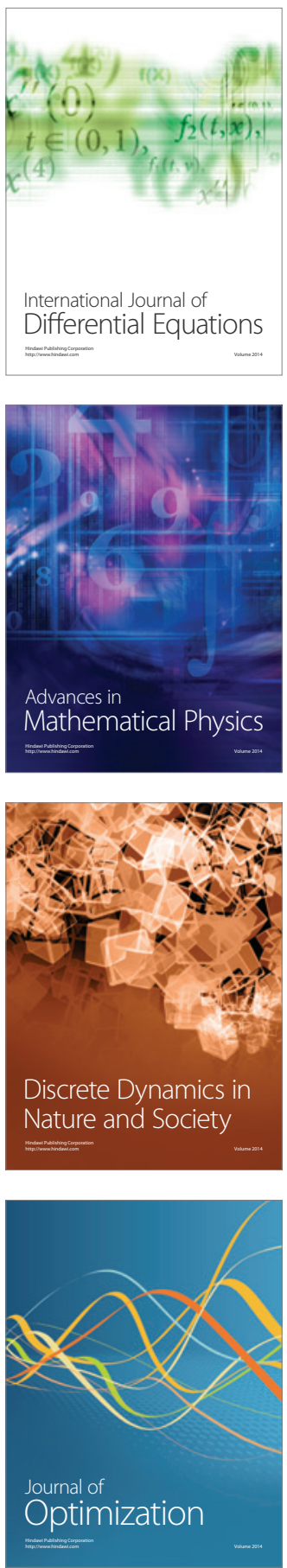